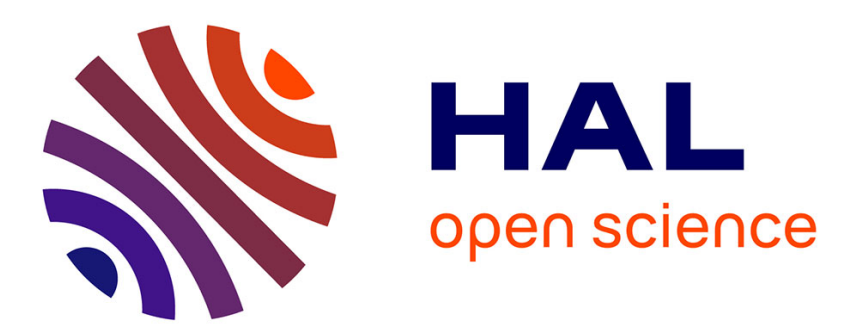

\title{
Formalising Information Scoring in a Multivalued Logic Framework
}

\author{
Adrien Revault d'Allonnes, Marie-Jeanne Lesot
}

\section{To cite this version:}

Adrien Revault d'Allonnes, Marie-Jeanne Lesot. Formalising Information Scoring in a Multivalued Logic Framework. 15th International Conference on Information Processing and Management of Uncertainty in Knowledge-Based Systems, IPMU2014, Jul 2014, Montpellier, France. pp.314-324, 10.1007/978-3-319-08795-5_33. hal-01215584

\section{HAL Id: hal-01215584 \\ https://hal.science/hal-01215584}

Submitted on 25 Oct 2017

HAL is a multi-disciplinary open access archive for the deposit and dissemination of scientific research documents, whether they are published or not. The documents may come from teaching and research institutions in France or abroad, or from public or private research centers.
L'archive ouverte pluridisciplinaire HAL, est destinée au dépôt et à la diffusion de documents scientifiques de niveau recherche, publiés ou non, émanant des établissements d'enseignement et de recherche français ou étrangers, des laboratoires publics ou privés. 


\title{
Formalising Information Scoring in a Multivalued Logic Framework
}

\author{
Adrien Revault d'Allonnes ${ }^{1}$ and Marie-Jeanne Lesot ${ }^{2,3}$ \\ 1 Université Paris 8, EA 4383, LIASD, FR-93526, Saint-Denis, France \\ Allonnes@ai.univ-paris8.fr \\ 2 Sorbonne Universités, UPMC Univ Paris 06, UMR 7606, \\ LIP6, F-75005, Paris, France \\ 3 CNRS, UMR 7606, LIP6, FR-75005, Paris, France \\ Marie-Jeanne.Lesot@lip6.fr
}

\begin{abstract}
This paper addresses the task of information scoring seen as measuring the degree of trust that can be invested in a piece of information. To this end, it proposes to model the trust building process as the sequential integration of relevant dimensions. It also proposes to formalise both the degree of trust and the process in an extended multivalued logic framework that distinguishes between an indifferent level and the actual impossibility to measure. To formalise the process, it proposes multivalued combination operators matching the desired behaviours.
\end{abstract}

Keywords: trust, information quality, many-valued logic, information scoring, trust building

\section{Introduction}

Amongst the diverse facets of information scoring, trust holds a central role. Qualifying the degree of trust that can be put in a piece of information can mean either evaluating its certainty - i.e. the reality of the fact it reports [1-3] or the extent to which the rater is convinced, based on the process by which he forms an opinion about a hitherto unknown piece of information $[4,5]$.

Existing models differ in the dimensions they take into account to assess the degree of trust but also in the formal paradigms they use to represent it. Among the existing criteria, some examples are reliability, competence, sincerity, intention of the source, credibility, understood as confirmation by other sources, or plausibility, defined as likelihood with respect to a priori knowledge of the piece of information [1-6].

Information scoring as evaluation of trust has been formalised using both symbolic and numeric frameworks to evaluate each dimension and combine them using various aggregation operators to yield the final degree of trust. The original military model uses two discrete graded scales, respectively measuring source reliability and information credibility [1]. The aggregation is simply a concatenation of the two resulting symbols. This leads to difficulty in comparisons, hindering the evaluation's legibility [7]. Other models present the degree of trust 
as a single numerical value, a coefficient between 0 and 1 , in either possibility [3] or evidence $[6,8,9]$ theory frameworks. In the former, for instance, the dimensions are evaluated using possibility distributions, their aggregation relies on discounting, conjunctive and reinforcement operators [3].

This paper proposes to formalise information scoring in an extended multivalued logic framework: first off, as in the numerical approaches mentioned above, a unique degree is returned; second, as in the symbolic approach, it is defined on a discrete graded scale, which is further clarified with linguistic labels specifying the degrees' meaning, for all measured quantities [5]. Indeed, multivalued logic offers formal tools and concepts [10] which allow these improvements of the model's legibility. The proposed extension of the multivalued framework arises in the introduction of an additional degree modelling the case where a dimension cannot be quantified, through lack of knowledge, thus distinguishing it from the case where the evaluation leads to a neutral, indifferent value.

Each dimension mentioned above is individually evaluated in this extended multivalued framework. The resulting level of trust is also measured in this framework by successive integration of the dimensions: this paper proposes to model the trust building process as the sequential projection of the activation along selected dimensions on the current level of trust. To achieve this, the required properties for information scoring process projection operators are discussed and corresponding functions are proposed.

The paper is organised as follows: Section 2 presents the trust building process as the sequential projection of dimensions on the level of trust. Section 3 presents the proposed extended multivalued logic framework that distinguishes between lack of knowledge and indifference. Sections 4 and 5 are dedicated to the two types of combination operators identified. The paper then concludes with some directions for future work.

\section{A Model of the Trust Building Process}

This section presents a general model of the process through which trust in a piece of information is constructed. The principle of this model lies in updating the trust value by the sequential projection of the dimensions which partake in its evaluation. In particular, this section focuses on the attributes which will be formalised in the following sections, such as the influence of dimension projection, leading to specifications for the projection operators, as well as the difference between unknown and neutral levels.

\subsection{Trust Building as a Sequence of Projections}

The model described in this paper dynamically measures trust as a score whose value shifts with the consecutive consideration of different dimensions. These factors are projected on the current score, resulting in an updated trust value: trust-building is modelled as the consecutive integration of answers to different questions. This principle is illustrated in Figure 1, discussed further below, where 


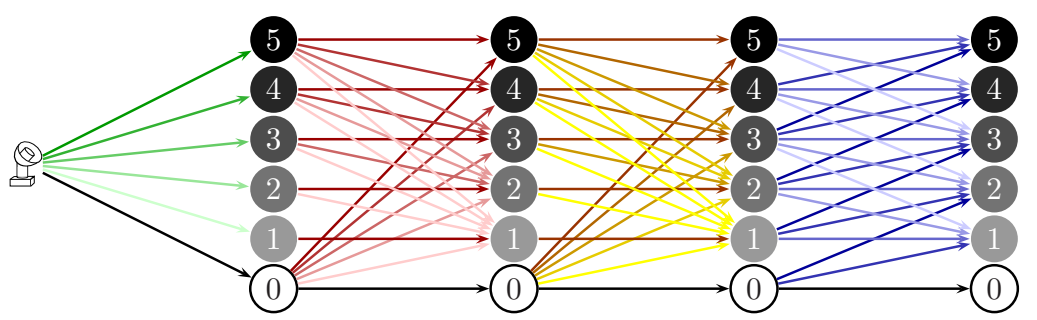

Fig. 1. Sequential projection of dimensions on trust

the shaded disks represent the current level of trust and the arrows four factors consecutively influencing its evolution. Obviously, the questions should not be redundant, nor should they be dependent. They should span different levels of specificity, from the most general, that is depending only on the context, not on the considered piece of information, to the particular, directly dependent on the informational content.

This principle is put in use in [5], where the dimensions are selected so as to consecutively answer the following questions: 'Who is telling me?', which corresponds to source reliability; 'What does he know about the topic?', to source competence; 'How likely does it seem?', to information plausibility; 'Is anyone else reporting it?', to information credibility. The first two relate to the source where the other two depend on the informational content. Furthermore, the evaluation of the source's reliability, how trustworthy the source is, is constant, whatever the considered piece of information, where competence depends both on the source and the topic, so is more specific to the piece of information. Plausibility, taken here as compatibility with the rater's knowledge, is contextual and subjective in that it depends both on the piece of information and the rater. Credibility is completely contextual, since it is taken as a degree of confirmation of the considered piece of information. The four dimensions therefore span the divide from source to information, general to contextual and subjective to objective, ensuring they are neither redundant nor dependent.

\subsection{Combining the Dimensions: Influence of the Projections}

Once the dimensions participating in the evaluation are selected, one must look at how their intensities are combined and the order in which they are considered. In order to model the trust building process, we propose to order the dimensions from the most immutable to the least, that is from the most general to the most specific, both content and contextwise. The idea behind this ordering is that the evaluation of trust starts with global dimensions and is progressively corrected with respect to the specifics of the considered piece of information.

These consecutive corrections entail that most projections have an abating influence. For instance, the model presented in [5] and briefly summarised above suggests that the evaluation starts with source reliability, the most general dimension as its value stays the same regardless of the content. It is then adapted 
to the assertion by the projection of source competence, this adaptation resulting in either a lower updated value or, at most, leaving it unchanged: faced with a trustworthy source, the fact that he is a specialist on the topic should not increase one's trust. Rather, if he were to talk about something which he knew nothing about, would one's doubts begin to rise. Similar arguments also apply to information plausibility. For this reason, an abating operator is needed to project most dimensions onto trust.

However, a second type of operator is necessary to model credibility, as this particular dimension can have both an increasing and weakening influence on the trust level. Indeed, the final step in a trust building process is to try to cross-check the informational content, i.e. to find confirmation or invalidation. Credibility appears in most existing models $[1,3,5]$, usually, as the final projected dimension. This is in agreement with the specificity ordering proposed above, as cross-checking is both content and context dependent. Another argument for the credibility being the final step in the process is that its projection depends on the score of the confirmation which, thus, needs to have already been computed. Regarding credibility's influence, confirmations increase the trust level where invalidations weaken it, which explains the need for a specific cross-checking operator rather than the abating operator which, by definition, is one-way.

These two types of operators are illustrated in Figure 1, where the colour intensity of each arrow corresponds to the level to which each dimension is measured. The second and third sets of arrows, which all - except for the ones coming from the disks labelled 0 , discussed in the following subsection - point downwards, are examples of abating operators. The final set of arrows, in both directions, is an example of the cross-checking operator.

\subsection{Preserving the Expressiveness of Ignorance}

The proposed sequential process relies on evaluations being made. However, there may be situations in which it is impossible to measure a particular dimension. It may, for instance, be impossible to evaluate an as yet unknown source's reliability, for lack of knowledge. Such a situation should not be confused with one in which a source is 'neither trustworthy nor untrustworthy', which conveys some knowledge on the matter. The latter is usually in the middleground of the evaluation scale. Distinguishing the two cases is necessary when projecting dimensions, as ignorance should not beget knowledge: if a dimension cannot be measured, its projection should not influence the outcome.

For this reason, a specific level is used in [1], representing the impossibility to measure. Likewise, in the example shown in Figure 1, this partly accounts for the horizontal arrows: projection of an impossible to measure dimension does not change the score, regardless of its current value. In addition, the disks labelled 0 represent a situation where the level of trust is unknown, i.e. none of the previous dimensions have been measured. In the absence of knowledge, the first dimension which can be evaluated moves the score out of ignorance. Note that, if evaluation of trust was possible at any stage, it remains possible in all subsequent stages. Thus, no arrow points to a disk marked 0 . 


\section{Extended Multivalued Logic Framework}

The multivalued logic paradigm is based on a symbolic, discrete truth scale and offers a formal framework to manipulate and combine these degrees [10]. It constitutes a candidate framework to model the trust building process described in the previous section, addressing the clarity and legibility concerns: the discrete scales used both for measuring the dimensions and the level of trust, such as that presented in Figure 1, can be mapped to multivalued logic degrees. Moreover, multivalued logic combination operators can help express trust as a projection of dimensions in a single degree. However, to fully express all aspects of the model, the multivalued logic paradigm needs to be able to distinguish between an indifferent and an unknown degree.

After having recalled the essential characteristics of multivalued logic, this section describes the proposed extension and discusses its properties.

\subsection{Multivalued Logic}

Multivalued logic models reasoning using the $M$ truth degrees of a totally ordered set $\mathcal{L}_{M}=\left\{\tau_{0}, \ldots, \tau_{M-1}\right\}$, where $\tau_{\alpha} \leq \tau_{\beta} \Leftrightarrow \alpha \leq \beta$, coupled to an involutive negation satisfying De Morgan's laws. The ordering property guarantees all degrees in $\mathcal{L}_{M}$ are comparable. They span, at a granularity varying with $M$, the different levels of veracity they represent from $\tau_{0}$, meaning 'false', to $\tau_{M-1}$, for 'true'. The switch between the two appears around the middle value $\tau_{\frac{M-1}{2}}$, usually forced into the chosen scale by choosing an odd $M$. Furthermore, all $\tau_{\alpha}^{2}$ 's come with a semantic label increasing their legibility.

On top of the expressiveness and legibility of the truth scale, multivalued logic offers tools to reason with truth degrees, through operators that generalise conjunction, disjunction or implication [10]. It also has operators for symbolic arithmetic, beyond a purely logic interpretation [11].

\subsection{Introduction of an Ignorance Degree: $\tau_{\text {? }}$}

Discrete scales used for information scoring can be mapped to multivalued logic degrees. In accordance with what was discussed in the previous section and in Section 2.3 , the indifferent value can be mapped to $\tau_{\frac{M-1}{2}}$, leaving the question of representing the absence of knowledge.

We propose to extend the multivalued logic framework by introducing an additional degree, denoted $\tau_{\text {? }}$, in order to distinguish between ignorance and indifference. We propose to define this degree by the following properties, where $\odot$ denotes any multivalued operator:

$$
\begin{array}{ll}
\text { 1. } \tau_{\text {? }} \notin \mathcal{L}_{M} & \text { 3. } \neg \tau_{?}=\tau_{?} \\
\text { 2. } \forall \tau_{\alpha} \in \mathcal{L}_{M}, \tau_{?} \odot \tau_{\alpha}=\tau_{\alpha} \odot \tau_{?}=\tau_{\alpha} & \text { 4. } \tau_{?} \odot \tau_{?}=\tau_{?}
\end{array}
$$

The first property sets $\tau_{\text {? }}$ apart from other degrees, not submitting it to the same ordering constraints as $\tau_{\alpha} \in \mathcal{L}_{M}$ : an element whose truth cannot be evaluated 
cannot be compared to an element whose truth is known, no matter how true. Therefore, we propose to define $\tau_{\text {? }}$ as an exception to the total order rule and of a different nature than other degrees. The second property defines $\tau_{\text {? }}$ as a neutral element for all operators, in particular for both conjunctions and disjunctions, which implies it does not preserve the order on $\mathcal{L}_{M}$. The last two properties define the behaviour of $\tau_{\text {? }}$ when combined with itself.

It can be shown easily that $\tau$ ? preserves some essential properties of a De Morgan algebra, specifically the involution of negation and De Morgan's laws, but contradicts the axioms of identity, complement as well as the absorption law.

\subsection{Characteristics of the Extended Multivalued Framework}

We propose that truth be evaluated on $\mathcal{L}_{M}$ extended with $\tau_{\text {? }}$, which we write $\mathcal{L}_{M}^{e}=\mathcal{L}_{M} \cup\left\{\tau_{\text {? }}\right\}$. This section shows how it offers an essential gain in expressiveness, illustrating it in the information scoring application.

As already stated, $\tau_{\text {? }}$ is not comparable to any degree, which may seem contradictory with the sought qualities of truth degrees. However, the localised loss of comparability in $\mathcal{L}_{M}^{e}$ is not an issue as $\tau_{\text {? }}$ never needs be compared to any degree, and, in fact, satisfies an expectation: a fact whose truth is given as $\tau_{\text {? }}$ is neither more, nor less true than any other. For this same reason, the introduction of $\tau_{\text {? }}$ places the comparison between a 'half true' fact and another back in its rightful place in $\mathcal{L}_{M}$ 's semantic hierarchy.

When constructing or modifying a logic framework, one should take heed of the impact on the consistency of the resulting system, i.e. the impossibility to infer a contradiction. In the case of multivalued logics, this property is already relaxed since the laws of excluded middle and noncontradiction do not hold. Now adding $\tau_{\text {? }}$ does not introduce inconsistency: because $\tau_{\text {? }}$ is defined as a neutral element for all combination operators, any inconsistency proved after its inclusion will persist should it be removed.

\section{Multivalued Abating Combination Operator}

The previous section defined the extended formal framework $\mathcal{L}_{M}^{e}$. This section and the next propose operators for manipulating degrees in $\mathcal{L}_{M}^{e}$ and, more specifically, operators exhibiting the properties detailed in Section 2 to model trust building. These operators are mappings $\mathcal{L}_{M}^{e} \times \mathcal{L}_{M}^{e} \longrightarrow \mathcal{L}_{M}^{e}$ with current score and evaluation of the dimension for inputs and updated score for output.

This section focuses on the abating operator whose necessity is detailed in Section 2. A formal description of the required properties is given before a corresponding function is defined.

\subsection{Formal Description of the Required Behaviour}

The properties needed for an abating operator $F: \mathcal{L}_{M}^{e} \times \mathcal{L}_{M}^{e} \longrightarrow \mathcal{L}_{M}^{e}$ are:

$$
\text { - } \forall \tau_{\alpha}, \tau_{\beta} \in \mathcal{L}_{M} F\left(\tau_{\alpha}, \tau_{\beta}\right) \leq \tau_{\alpha}
$$


$-\forall \tau_{\alpha} \in \mathcal{L}_{M}^{e} \quad F\left(\tau_{?}, \tau_{\alpha}\right)=F\left(\tau_{\alpha}, \tau_{?}\right)=\tau_{\alpha}$

- $\forall \tau_{\alpha}, \tau_{\beta}, \tau_{\gamma} \in \mathcal{L}_{M}$ if $\tau_{\alpha} \leq \tau_{\beta}$, then $F\left(\tau_{\alpha}, \tau_{\gamma}\right) \leq F\left(\tau_{\beta}, \tau_{\gamma}\right)$ and $F\left(\tau_{\gamma}, \tau_{\alpha}\right) \leq F\left(\tau_{\gamma}, \tau_{\beta}\right)$

The first property guarantees the abating behaviour, by imposing the updated value is at most equal to the current value $\tau_{\alpha}$. The second property defines $\tau_{\text {? }}$ as a neutral element of $F$ : when the current score is still unknown, the value of the projected dimension directly determines the updated score. Conversely, when the level of the dimension cannot be measured, the score is not updated. The final property ensures the operator is increasing in both its arguments. Indeed, the higher the current score, the higher the updated one, for any correction; reciprocally, for any given level of trust, the more the dimension is activated, the higher the updated score.

\subsection{Proposed Operator}

In order to satisfy the properties listed in the previous subsection, one could consider extending t-norms. Indeed, t-norms, in the regular multivalued case, satisfy the first and third properties, leaving only the special case of $\tau_{\text {? }}$. However, t-norms have other properties, such as commutativity and associativity, which are superfluous in this particular context and would lead to unwanted constraints, limiting the expressiveness of the model. Furthermore, even if these properties were accepted, the different nature of the arguments, current score vs. dimension level, makes them inconsistent.

We, therefore, propose to define a new operator, illustrated in Figure 2, based on a set of parameters $\kappa_{\alpha}^{\gamma} \in \mathcal{L}_{M}$ that define transition thresholds between a current score $\tau_{\alpha}$ and its updated value $\tau_{\gamma}$ :

$$
F\left(\tau_{\alpha}, \tau_{\beta}\right)= \begin{cases}\min \left\{\tau_{\gamma} \in \mathcal{L}_{M} \mid \tau_{\beta} \leqslant \kappa_{\alpha}^{\gamma}\right\} & \text { if } \tau_{\alpha}, \tau_{\beta} \in \mathcal{L}_{M} \\ \tau_{\beta} & \text { if } \tau_{\alpha}=\tau_{?} \\ \tau_{\alpha} & \text { if } \tau_{\beta}=\tau_{?}\end{cases}
$$

The first line sets the conditions for the transition from $\tau_{\alpha}$ to $\tau_{\gamma}$. The other cases describe $\tau_{\text {? }}$ as a neutral element for both arguments.

Note that consistency with the proposed model imposes constraints on the parameters $\kappa_{\alpha}^{\gamma}$ : to guarantee the function is increasing, they must be ordered, so that if $\tau_{\gamma} \leq \tau_{\delta}$, then $\kappa_{\alpha}^{\gamma} \leq \kappa_{\alpha}^{\delta}$. However, these ordering constraints on $\kappa_{\alpha}^{\gamma}$ are imposed for a given value of $\tau_{\alpha}$ but constraints of the form $\kappa_{\alpha}^{\beta}=\kappa_{\alpha}^{\gamma} \oplus \kappa_{\gamma}^{\beta}$, where $\tau_{\gamma}$ is an intermediate value between $\tau_{\alpha}$ and $\tau_{\beta}$ and $\oplus$ a suitable sum operator [11], are not required: going directly from $\tau_{\alpha}$ to $\tau_{\beta}$ need not be equivalent to successive transitions from $\tau_{\alpha}$ to $\tau_{\gamma}$ and from $\tau_{\gamma}$ to $\tau_{\beta}$.

\section{Multivalued Cross-checking Combination Operator}

Two remarkable properties of credibility projection require a dedicated operator: first it can both weaken or increase the level of trust. Second, it involves an additional argument: besides the usual current score and dimension level, it 


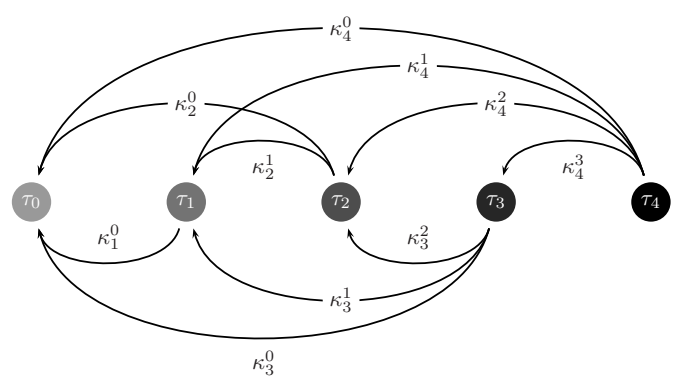

Fig. 2. An example of $F\left(\tau_{\alpha}, \tau_{\beta}\right)$ ignoring $\tau_{\text {? }}$ : the degrees of $\mathcal{L}_{5}$ represent possible values of the current score, the arrows labelled with $\kappa_{\alpha}^{\gamma}$ transitions from $\tau_{\alpha}$ to $\tau_{\gamma}$

depends on the current evaluation of the confirmation or invalidation. Indeed, an absolute confirmation should obviously increase the level of trust. However, if said corroboration has a low trust value, its influence should be less than that of a highly trusted confirmation.

\subsection{Formal Description of the Required Behaviour}

The cross-checking operator $G$ is therefore a mapping, $G: \mathcal{L}_{M}^{e} \times \mathcal{L}_{M}^{e} \times \mathcal{L}_{M}^{e} \longrightarrow$ $\mathcal{L}_{M}^{e}$, satisfying the following properties:

- $G$ is increasing in all 3 arguments

- $\tau_{\text {? }}$ is a neutral element for all 3 arguments

- $\forall \tau_{\alpha}, \tau_{\beta}, \tau_{\gamma} \in \mathcal{L}_{M} G\left(\tau_{\alpha}, \tau_{\beta}, \tau_{\gamma}\right) \geq \tau_{\alpha}$ if $\tau_{\beta} \geq \tau_{\frac{M-1}{2}}$

$G\left(\tau_{\alpha}, \tau_{\beta}, \tau_{\gamma}\right) \leq \tau_{\alpha}$ if $\tau_{\beta}<\frac{\tau_{\frac{M-1}{2}}^{2}}{2}$

The arguments for the monotonicity of operator ${ }^{2} G$ are similar to the ones explaining F's, as given in Section 4.1. The third property outlines the change of behaviour between confirmations (where credibility is in the top half of the scale: $\tau_{\beta} \geq \tau_{\frac{M-1}{2}}$ ), for which $G$ exhibits an increasing trend, and invalidations which have a weakening effect on the trust level.

\subsection{Proposed Operator}

Based on the properties required of the cross-checking operator, we propose the following function, illustrated in Figure 3:

$$
G\left(\tau_{\alpha}, \tau_{\beta}, \tau_{\gamma}\right)= \begin{cases}\tau_{\alpha} & \text { if } \tau_{\beta}=\tau_{?} \\ \tilde{F}_{i}\left(\tau_{\alpha}, g\left(\tau_{\beta}, \tau_{\gamma}\right)\right) & \text { if } \tau_{\beta}<\tau_{\frac{M-1}{2}} \\ \tilde{F}_{c}\left(\tau_{\alpha}, g\left(\tau_{\beta}, \tau_{\gamma}\right)\right) \text { otherwise }\end{cases}
$$

As detailed below, $G$ 's increasing and weakening trends are described by functions $\tilde{F}_{c}$ and $\tilde{F}_{i}$ respectively.

In both cases, the range of influence is determined by a weighted credibility, defined as the aggregation of the degree of confirmation/invalidation, as measured by the credibility dimension $\tau_{\beta}$, and the trust level of the cross-checking 


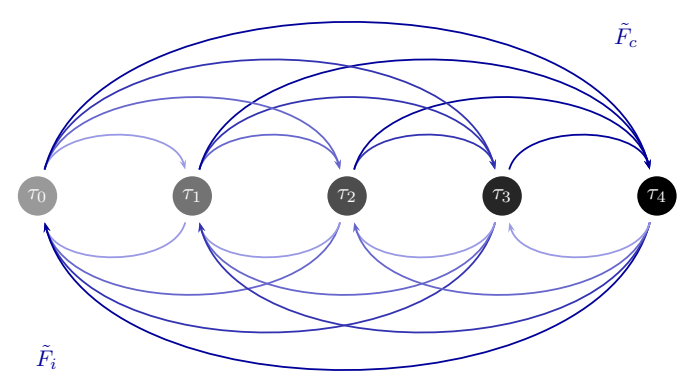

Fig. 3. An example of $G, \tau_{\beta}\left(\tau_{\alpha}, \tau_{\gamma}\right)$, in $\mathcal{L}_{5}$

piece of information $\tau_{\gamma}$. The aggregation operator $g: \mathcal{L}_{M}^{e} \times \mathcal{L}_{M}^{e} \longrightarrow \mathcal{L}_{M}^{e}$ must satisfy the following boundary conditions:

$$
\begin{array}{rlrl}
\forall \tau_{\alpha}, \tau_{\beta} \in \mathcal{L}_{M} g\left(\tau_{\alpha}, \tau_{\beta}\right) & \leq \tau_{\alpha} & \text { if } \tau_{\alpha} \geq \tau_{\frac{M-1}{2}} \\
g\left(\tau_{\alpha}, \tau_{\beta}\right) \leq \neg \tau_{\alpha} & \text { if } \tau_{\alpha}<\tau_{\frac{M-1}{2}}
\end{array}
$$

Indeed, the trust level of a confirmation can only weaken the impact of the credibility projection, along the same lines as the abating operator. The case of invalidation is similar, except for the negation of the credibility, which only serves to allow a symmetric behaviour. The monotonicity of $g$ must satisfy the following constraints, whose formal description is omitted for lack of space: $g$ must be increasing (resp. decreasing) in its first argument for confirmations (resp. invalidations); $g$ must be increasing in its second argument. Finally, $\tau_{\text {? }}$ must be an absorbing element for both arguments: if either cannot be evaluated, neither can the weighted credibility.

The $\tilde{F}_{i}$ operator, which controls the abating influence of weighted invalidations, is similar to the general abating operator defined in Section 4, its only specificity being its monotonicity in its second argument. Indeed, $\tilde{F}_{i}\left(\tau_{\alpha}, \tau_{\beta}\right)$ increases whereas $F\left(\tau_{\alpha}, \tau_{\beta}\right)$ decreases, when $\tau_{\beta}$ grows. Consequently, constraints on $\kappa_{\alpha}^{\gamma}$, and their comparisons with $\tau_{\beta}$, are reversed in $\tilde{F}_{i}$ defined similarly to $F$ :

$$
\tilde{F}_{i}\left(\tau_{\alpha}, \tau_{\beta}\right)= \begin{cases}\min \left\{\tau_{\gamma} \in \mathcal{L}_{M} \mid \tau_{\beta} \geq \kappa_{\alpha}^{\gamma}\right\} & \text { if } \tau_{\alpha}, \tau_{\beta} \in \mathcal{L}_{M} \\ \tau_{\alpha} & \text { if } \tau_{\beta}=\tau_{?} \\ \tau_{\beta} & \text { if } \tau_{\alpha}=\tau_{?}\end{cases}
$$

An example of such an operator is represented in the bottom half of Figure 3.

The $\tilde{F}_{c}$ operator, which controls the emphasising influence of weighted confirmations, satisfies properties similar to that of $F$, except for the first one which becomes $\forall \tau_{\alpha}, \tau_{\beta} \in \mathcal{L}_{M}, \tilde{F}_{c}\left(\tau_{\alpha}, \tau_{\beta}\right) \geq \tau_{\alpha}$, to express the emphasising influence. We thus propose to define:

$$
\tilde{F}_{c}\left(\tau_{\alpha}, \tau_{\beta}\right)= \begin{cases}\max \left\{\tau_{\gamma} \in \mathcal{L}_{M} \mid \tau_{\beta} \geq \kappa_{\alpha}^{\gamma}\right\} & \text { if } \tau_{\alpha}, \tau_{\beta} \in \mathcal{L}_{M} \\ \tau_{\alpha} & \text { if } \tau_{\beta}=\tau_{?} \\ \tau_{\beta} & \text { if } \tau_{\alpha}=\tau_{?}\end{cases}
$$


An example of such an operator is represented in the top half of Figure 3. Note that the definitions of the $\tilde{F}_{i}$ and $\tilde{F}_{c}$ functions are independent, it is not required that $\kappa_{\alpha}^{\gamma}=\kappa_{\gamma}^{\alpha}$ to allow for different sentivities to contradictory arguments.

\section{Conclusion and Future Works}

This paper proposes a sequential model for a trust building process, as the consecutive projection of dimensions on the current evaluation, as well as its formal and operational transposition in an extended multivalued logic framework. The latter offers a way to distinguish between indifference and ignorance, which is critical to adequately represent trust building. From the characterisation of the desired properties for the required projection operators, it defines special combination operators and gives the formal expression of suitable functions.

Future works will include the implementation of the proposed model and the definition of an evaluation protocol to lead an experimental study of user response to the proposed model. On a more formal level, another perspective lies in the theoretical study of the logical reasoning properties offered by the proposed extended multivalued logic $\mathcal{L}_{M}^{e}$, beyond the degree manipulation tools explored in this paper. Regarding information scoring, future works will aim at proposing tools allowing for the integration of the dynamics of trust building, like the influence on the evolution of trust of alternating confirmations and invalidations.

\section{References}

1. NATO: Annex to STANAG2022. Information handling system (1997)

2. Demolombe, R.: Reasoning about trust: a formal logical framework. In: Int. Conf. on iTrust. (2004)

3. Lesot, M.J., Delavallade, T., Pichon, F., Akdag, H., Bouchon-Meunier, B.: Proposition of a semi-automatic possibilistic information scoring process. In: Proc. of EUSFLAT'11. (2011) 949-956

4. Besombes, J., Revault d'Allonnes, A.: An extension of STANAG2022 for information scoring. In: Proc. of the Int. Conf. on Information Fusion. (2008) 1635-1641

5. Revault d'Allonnes, A.: An architecture for the evolution of trust: Definition and impact of the necessary dimensions of opinion making. In Capet, P., Delavallade, T., eds.: Information Evaluation. Wiley (2014) 261-292

6. Pichon, F., Dubois, D., Denœux, T.: Relevance and truthfulness in information correction and fusion. IJAR 53(2) (2011) 159-175

7. Capet, P., Revault d'Allonnes, A.: Information evaluation in the military domain: Doctrines, practices and shortcomings. In Capet, P., Delavallade, T., eds.: Information Evaluation. Wiley (2014) 103-128

8. Cholvy, L.: Information evaluation in fusion: a case study. In: Proc. of IPMU'04. (2004) 993-1000

9. Cholvy, L.: Evaluation of information reported: a model in the theory of evidence. In: Proc. of IPMU'10. (2010) 258-267

10. Akdag, H., De Glas, M., Pacholczyk, D.: A qualitative theory of uncertainty. Fundamenta Informaticae 17(4) (1992) 333-362

11. Seridi, H., Akdag, H.: Approximate Reasoning for Processing Uncertainty. J. of Advanced Comp. Intell. and Intell. Informatics 5(2) (2001) 110-118 